\title{
Body Temperature Patterns in Captive Barbary Striped Grass Mousse (Lemniscomysbarbarus): Influence of Photoperiod and Sex Steroids
}

\author{
Malika Nait Daoud, Ali Ouarour \\ Laboratory of Biology and HealthUniversityAbdelmalekEssaâdi Tétouan, Morocco \\ Malika-nd@live.com
}

\begin{abstract}
The Barbary striped grass mouse (Lemniscomys Barbarus) has been described as a diurnal and photoperiodic rodent, but no reliable data exist on the daily and seasonal rhythmicity of body temperature (Tb). In this study, Tb patterns were determined in different groups of males maintained in captivity under different regimes of photoperiod. Tb was recorded with surgically implanted iButton temperature loggers. The work shows the existence of a strong and bimodal daily Tb rhythm in this species whose external synchronizer is the light-dark cycle; it's also confirmed the diurnal and photoperiodic character of this animal. The highest values of Tb were registered during the light-phase in the contrary of nocturnal species. This kind of experimental work corresponds to the theme of the ACMLIS'17 conference by means of, on one side, the use of the temperature-sensitive intra-abdominal implants for monitoring core body temperature, an approach of machine learning, and on the other side, the final aim of the work which is to better understand the more complicated and sophisticated of intelligent systems, the central nervous system.
\end{abstract}

Keywords-body temperature; daily torpor; photoperiod; light-dark cycle; diurnality; Lemniscomys barbarus.

\section{Introduction}

In this research study, the daily body temperature $\left(T_{b}\right)$ variations following the light/dark cycle were measured on a long time. This kind of experimental works joins perfectly within the framework of the theme of the international conference on the emotional computing, learning machine and the intelligent systems (ACMLIS'17), on one hand, by the use of a technique of continuous measurement of core body temperature, and on the other hand, by their scientific objective to decipher the mechanisms of measurement of the notion of time by the central nervous system. Indeed, no better inspiration for the development of the affective computing as the one who would ensue from a better understanding the functioning of the brain of mammals, a structure of all the human feelings integration. Besides, the technique of the temperature-sensitive intra-abdominal implants for monitoring core body temperature (data loggers DS1922L Thermochron iButtons ${ }^{\circledR}$ ) is an approach of the machine learning since it allows an analysis of curves of temporal evolution of a biological measurement, in this particular case the continuous values of the body temperature. So, the definition of the intelligent systems, in a general 
framework, covers perfectly the domain of sophisticated interfaces as can well it be the one of the temperature-sensitive intra-abdominal data loggers. The core body temperature-sensitive data loggers are capable of automatically recording temperature over a long defined period of time. They are small, battery powered, and equipped with a microprocessor, internal memories for data storage, and sensors. The sampling methods definition is performed by custom-made computer programs and the data are downloaded to the computer via an interface.

Furthermore, in mammals, daily rhythms in many biological processes are a fundamental feature that enables them to anticipate and acclimatize to predictable changes in environment factors. Expression of this rhythmicity with a period of approximately $24-\mathrm{h}$ (i.e. circadian) is orchestrated by the suprachiasmatic nucleus (SCN) of the anterior hypothalamus, site of the master endogenous circadian clock [15].

In mammals including humans, like to numerous birds, regular daily $T_{b}$ variations follow the light/dark cycle. A thermal peak is observed during the day in the diurnal animals and the opposite in the nocturnal ones. These cycles of $T_{b}$ remain in the absence of any external synchronizer; they are also physiological readout controlled by the SCN [42]. The cost of maintaining high body temperatures in endothermic animals is high. It constitutes a substantial component of their resting metabolic rate and is directly related to ambient temperature [25].

Many small mammals live "right on the edge" with the amount of food that they eat each day barely sufficient to keep them alive and warm until morning. As a result, added stresses on the animal, particularly seasonal shortages of food/water and/or extreme environmental temperatures can be lethal. The solution for many small mammals is to temporarily lower their energy needs by regulating a strong suppression of their metabolic rate, causing $T_{b}$ fall, and enter either short-term daily torpor or long-term continuous hibernation $[18 ; 20 ; 33 ; 9 ; 2]$. Torpor involves the regulation of $\mathrm{T}_{\mathrm{b}}$ at a new and substantially lower level, with a new minimum $\mathrm{T}_{\mathrm{b}}$ being maintained [8]. Daily torpor lasts for only a matter of hours each day, usually interrupted by periods of diurnal foraging and feeding [19; 43; 12].

Daily $T_{b}$ rhythm seems to be correlates with the period of activity; the locomotor activity is undoubtedly the best-studied daily rhythmic output of the circadian pacemaker. Concerning this pattern, a difference exists between diurnal and nocturnal organisms because the first ones are active mostly during the day while the second ones are active mostly during the night. The neural mechanisms responsible for diurnality or nocturnality are not well identified [46; 7].

Much less chronobiological studies concern $\mathrm{T}_{\mathrm{b}}$ rhythms in diurnal species (e.g., Octodon degus, [23]; Arvicanthis niloticus, [27]; Arvicanthis ansorgei, [4; 41]). These studies, however, are important since diurnal models present similar temporal organization of the sleep/wakefulness rhythm to that of humans and as such, these animal models can be used for suitable biomedical applications. Recently, the Barbary striped grass mouse, Lemniscomys barbarus, has been proposed as a new diurnal rodent model for choronobiological studies. More precisely, the circadian organization of wheel-running activity rhythm and cytoarchitecture of the SCN have been described in this African diurnal rodent [21]. We know that Lemniscomys barbarus is a photoperiodic species because its transfer from long photoperiod (LP) to short photoperiod (SP) results in a small decrease in its gonadal activity [22].

The objective of the present work was to define better the circadian characteristics of this specific diurnal model by studying the $T_{b}$ daily rhythms in different photoperiodic regimes. In addition, we investigated 
Malika Nait Daoud, Ali Ouarour; Body Temperature Patterns in Captive Barbary Striped Grass Mousse (Lemniscomysbarbarus): Influence of Photoperiod and Sex Steroids. Transactions on Machine Learning and Artificial Intelligence, Vol 5 No 4 August (2017); pp: 30-42

possible role of sex steroids in seasonal changes of daily rhythm of $T_{b}$. Moreover, because the recording of $T_{b}$ was continuous throughout the experiment, we also check if daily torpor or hibernation bouts were expressed during prolonged exposure to SP conditions.

\section{Materials and Methods}

\subsection{Animals}

Barbary striped grass mouse, Lemniscomys barbarus (Linnaeus 1766) is a rodent (suborder: Sciurognathi; family: Muridae). This species has a strictly African distribution, and can be commonly found in North and sub-Saharan Africa in dry and semi-arid zones, where it frequents local biotopes relatively wet and rich in vegetation. The reproduction of this mammal appears to take place mainly in the spring/summer months (Ouarour, unpublished observations).

Since 2004 a breeding colony is developed at the Faculty of Science in Tétouan (north of Morocco). Animals used in the present study freshly trapped in the region of Tétouan around the village of Benkarrich (around $35^{\circ} 30 \mathrm{~N}, 5^{\circ} 25 \mathrm{~W}$ ). At this latitude, photoperiod changes from a maximum of $14 \mathrm{~h} 35$ to a minimum of $9 \mathrm{~h} 43$ of light. The annual climate presents mainly two distinct seasons: a raining or wet season from October to April and a drier season from May to September. Ambient temperature oscillates from a minimum during the mild winters of $3-8^{\circ} \mathrm{C}$ around January to a maximum during the relatively hot summer of $30-35^{\circ} \mathrm{C}$ around August.

In the area of captures, water is available even in summer. Every year, captures were made throughout the summer (June 15th to September 15th) with traps checked and baited daily with fresh food (bread and olive oil). After transport to the laboratory, trapped animals were housed singly in transparent cages (22x16x14 cm) under controlled squared 16h light-8h dark cycles (LD: 16/8, lights from 04:00 to 20:00 h, white light 200-300 lux during light phase, constant $<5$ lux red light). Ambient temperature was $22.2 \pm 2{ }^{\circ} \mathrm{C}$. Water and food (barley and pellets B3, Agropress, Casablanca, Morocco) were supplied ad libitum. Animals used in this work had a body weight ranging between 33 and $59 \mathrm{~g}$.

All manipulations of animals were made in Morocco in agreement with local legislation. Moreover, all procedures used in animal experimentation complied with the French National law, implementing the European Communities Council Directive 86/609/EEC. All efforts were made to minimize the number of animals used and their suffering, and the study met the ethical standards..

\section{Tb Recording}

Animals were anesthetized intraperitoneally by a mixture of $0.1 \mathrm{ml}$ of Rompun $2 \%$ (Xylazine) and $0.8 \mathrm{ml}$ of Zoletil 20 completed in a final volume of $5 \mathrm{ml}$ with the $\mathrm{NaCl} 0.9 \%(0.15 \mathrm{ml} / 10 \mathrm{~g}$ bodyweight). Anesthesia lasted more than one hour when implanting the miniature data loggers. Just before the injection of anesthetics we made the animal sniffing at cotton soaked with halothane (Laboratoires BELAMONT, Paris, FRANCE). A horizontal incision of less than $20 \mathrm{~mm}$ was made through the abdominal surface beforehand shaved in scissors and disinfected with alcohol solution and the data loggers were implanted. The incision was sutured closed and the wound sprayed with an antiseptic spray. After surgery, hypothermia was avoided with exposure in a heat coming from a light source. Generally, all animals recovered completely from implant surgery and their behavior it's normal. 
The temperature-sensitive data loggers (DS1922L Thermochron iButtons ${ }^{\circledR}$, Dallas Semiconductor, Texas, USA) used were coated by biocompatible silicone. The loggers were set to record the animals' Tb every 30 min with a resolution of $0.5^{\circ} \mathrm{C}$ and during 25 weeks. The data stored in i-buttons are got back at the end of the experiment via a reader connected directly with a computer by a bearing COM.

\section{Testosterone Administration and Castration}

Testosterone capsules (4-androsten-17 $\beta$-ol-3-one, Sigma, Saint-Louis, MO, USA) measuring $10 \mathrm{~mm}$ were prepared with silastic tubes (DOW Coming, Midland, MI, USA; $1.57 \mathrm{~mm}$ internal diameter and $2.4 \mathrm{~mm}$ external diameter). Their extremities were occluded with silastic glue (DOW Corning). Testosterone capsules were stored at $4^{\circ} \mathrm{C}$ until use. In the Siberian hamster, Phodopus sungorus, a rodent of the same size as our animal model, capsules of this size filled with testosterone reproduce the physiological plasmatic concentrations [53]. The subcutaneous implantation of capsules was made by making a small latero-dorsal incision in the same time that of implantation of data loggers. Castrations were also performed by an abdominal incision in the same time that of implantation of data loggers.

\section{Experimental Design}

To establish the daily pattern of Tb rhythm in Lemniscomys barbarus, the effects of photoperiod and sexual steroids on daily rhythm of Tb, a total of adult's 20 males, trapped between July and October, 2009, were implanted with data loggers at the first week of December, 2009. The animals were subdivided into various experimental groups for Tb continuous recording during 25 weeks until May, 2010:

- Males animals were maintained in LP: $16 / 8$ conditions ( $\hat{o}$ LP group; $n=5$ );

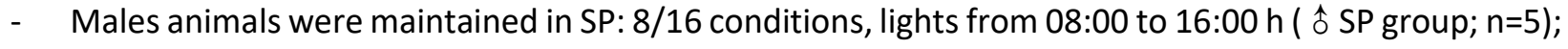

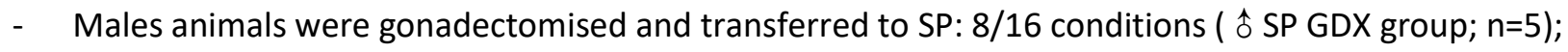

- Males animals were gonadectomised, implanted with testosterone capsules and transferred to SP:

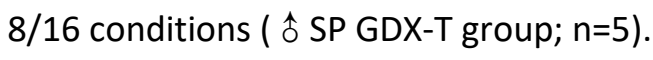

\section{Data Analysis}

All data are expressed as means \pm S.E.M. Tb measurements were averaged for each half hour. Circadian rhythms were assessed by cosinor analysis using a nonlinear regression model. The analysis generated the following rhythm descriptors: mean level, amplitude and acrophase of Tb with SigmaPlot 11 software (Jandel Scientific, Chicago II, USA). To take into account the bimodal pattern of body temperature in Lemniscomys barbarus, a much better fitting was obtained with double-peak cosinor regression [?6; 5? as follows:

$$
\begin{gathered}
\mathrm{f}=\mathrm{y} 0+\left(\left(\mathrm{a} /\left(\left(1+\exp \left(\operatorname{slope}^{*}(\mathrm{t} 1-\mathrm{d}-\mathrm{x})\right)\right) *\left(1+\exp \left(\text { slope }^{*}(-\mathrm{t} 1-\mathrm{d}+\mathrm{x})\right)\right)\right)\right)+\left(\mathrm{a} 2 /\left(\left(1+\exp \left(\text { slope }^{*}(\mathrm{t} 2-\mathrm{d}-\right.\right.\right.\right.\right. \\
\mathrm{x}))) *(1+\exp (\operatorname{slope} 2 *(-\mathrm{t} 2-\mathrm{d}+\mathrm{x}))))))
\end{gathered}
$$

Where $y 0$ is the mean level, a the amplitude, $t$ acrophase, $d$ half-duration to reach the peak, a 2 and $t 2$ are the secondary amplitude and acrophase of double peak. We explored changes in Tb using ANOVA; Two Way Analysis of Variance was performed with using of 'Bonferroni t-test' for all pairwise multiple comparison procedures. The effects are considered as significant if $p<0.05$. 
Malika Nait Daoud, Ali Ouarour; Body Temperature Patterns in Captive Barbary Striped Grass Mousse (Lemniscomysbarbarus): Influence of Photoperiod and Sex Steroids. Transactions on Machine Learning and Artificial Intelligence, Vol 5 No 4 August (2017); pp: 30-42

\section{Results}

\subsection{Daily patterns of Tb: photoperiodic effects}

The records of body temperature of a representative Lemniscomys barbarus maintained under a natural photoperiod are shown in figure (1-a). Clair daily rhythmicity is evident. Temperature oscillates between $36.41^{\circ} \mathrm{C}$ and $39.91^{\circ} \mathrm{C}$, corresponding to normothermia, with a mean of $37.83^{\circ} \mathrm{C}$. To facilitate visual inspection of the synchronization between the body temperature rhythm and the light-dark cycle, Figure (1-b) and (1-c) show the grand means of temperature calculated by first averaging the body temperature readings at each time point over the 10 days for each animal and then averaging the data for the animals.

The majority of the highest values of daily thermal activation were positioned during the light-Phase. The cosinor regression of the daily profiles of $T_{b}$ confirmed that the values variations are synchronized by the light-dark cycle (LD) as well in LP (Fig.1) as in SP conditions (Fig.2): the values of $T_{b}$ rose during the lightphase and their reduction coincided widely with the night-phase.

Profiles of the average daily $T_{b}$ rhythm corresponding to males exposed to LP (Fig.1) or SP (Fig.2) conditions show that the $T_{b}$ rhythm is biphasic independently of photoperiodic regime. In LP conditions, there was a morning peak at $4.07 \pm 0.24$ (Zeitgeber time) and later ones at $15.88 \pm 0.15$. Peaks occurred during the lightphase which extends from 04:00-20:00h.

In SP conditions, the first acrophase is at $2.46 \pm 0.48$ of dark-phase and the last one at $15.83 \pm 0.13$ in the end of light- phase. In SP conditions, lights from 08:00 to 16:00h show that the first acrophase took place during the dark-period while the second one occurred just before light-offset.

The average $T_{b}$ values in SP conditions was significantly higher in males in comparison to LP conditions (37.17 vs. 36.73, $p<0.001$; Bonferroni t-test). Nevertheless, the average of the first and second amplitude in LP were significantly higher than SP conditions (1.28 vs. $0.70, p<0.001 ; 3.17$ vs. $2.26, p<0.001)$.

The acrophase was significantly differences between LP vs. SP for the first and second peak, respectively, 5.19 vs. $3.57(p<0.001)$ and 15.08 vs. $15.02(p<0.001)$. The first and second acrophase were significantly phase advanced in SP conditions compared to LP. Acrophase was phase advanced in SP than LP conditions.

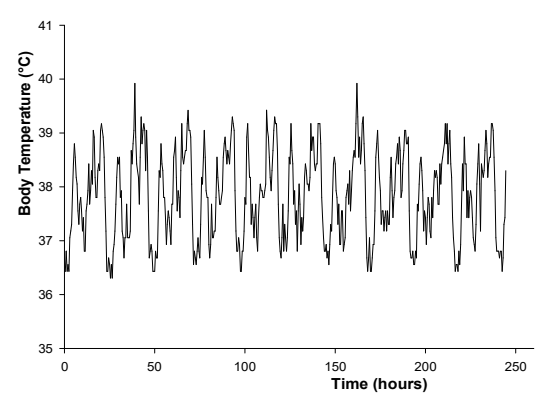

(a)

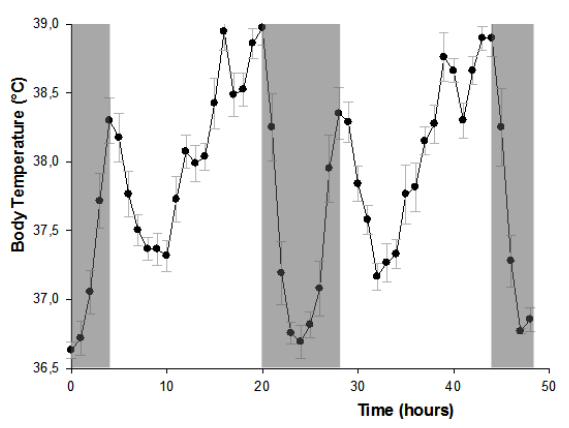

(b) 


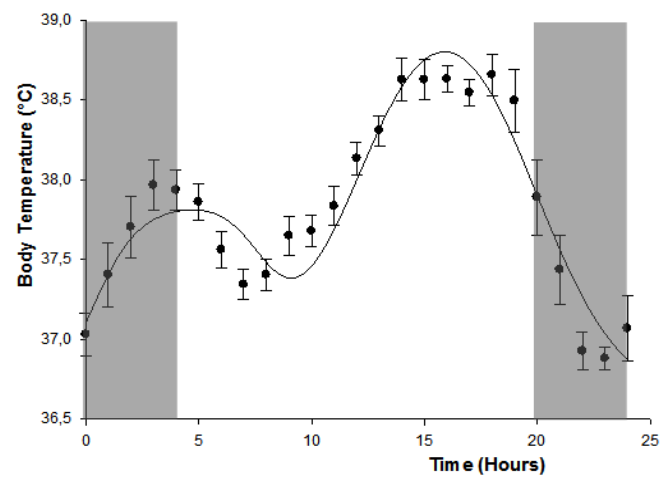

(c)

Figure 1. Body temperature $\left(\mathrm{Tb},{ }^{\circ} \mathrm{C}\right)$ of Lemniscomys barbarus males during exposure to a long photoperiod (LD 16:8). (a): continuous measurement of Tb over 10 consecutive days, (b): mean ( \pm SEM) body temperature, each averaged over 10 days, as function of time of day. (c): mean of Tb with cosinor regressions on 10 consecutive days of recording of Tb (-: express the best-fit non linear regressions). The grey shading indicates the dark phase.

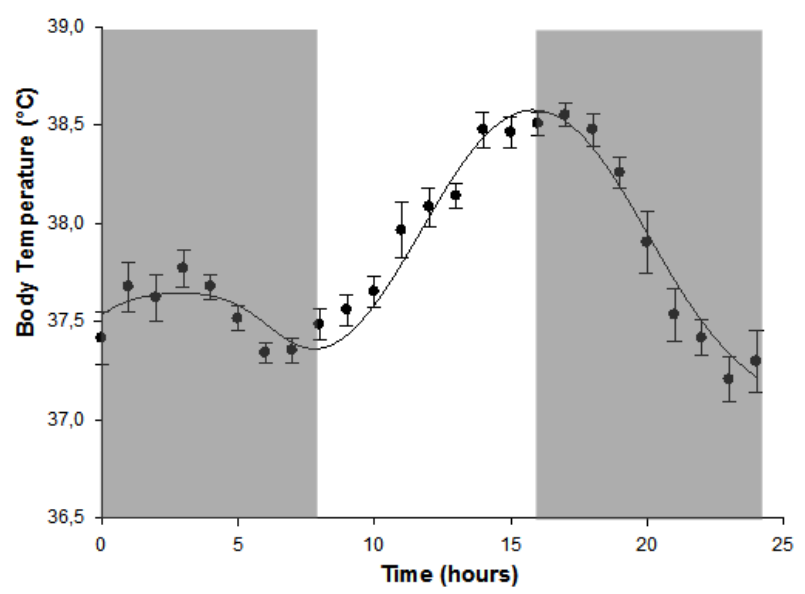

Figure 2. Daily profiles of the average of Tb rhythm in males Lemniscomys barbarus during exposure to a winter-like short photoperiod (LD 8:16). (-: express the best-fit non linear regressions). The grey shading indicates the dark phase.

\subsection{Daily patterns of Tb: photoperiodic effects}

In SP conditions, castrated animals showed a biphasic daily $T_{b}$ rhythm independently of testosterone administration or not (Fig. 3). In SP GDX group, the average value of $T_{b}$ on the 10 consecutive analyzed days of measurement was $37.26 \pm 0.05^{\circ} \mathrm{C}$, the first acrophase was at $3.92 \pm 0.22$ and the second one was at $14.05 \pm 0.13$. While the average for the first amplitude was $0.86 \pm 0.15$, the second one was $2.73 \pm 0.39$.

In SP GDX-T group, the average value of $\mathrm{T}_{\mathrm{b}}$ on the 10 analyzed days of measurement was $37.25^{\circ} \mathrm{C} \pm 0.12$, the first acrophase was at $4.34 \pm 0.48$ and the second one was at $15.20 \pm 0.19$. The average for the first amplitude was $0.58 \pm 0.11$ and $2.00 \pm 0.12$ for the second one.

The comparison of GDX group vs. control group (intact males) shows that the average value of $\mathrm{T}_{\mathrm{b}}$ was significantly higher in GDX group ( $p<0.001,37.04$ vs. 36.78; Bonferroni t-test). Also, the average of the first and second amplitude in GDX group were significantly higher than control group (1.16 vs. 0.94, $p<0.001 ; 3.18$ vs. $2.50, p<0.001)$. The first acrophase was significantly delayed in GDX group than control 
Malika Nait Daoud, Ali Ouarour; Body Temperature Patterns in Captive Barbary Striped Grass Mousse (Lemniscomysbarbarus): Influence of Photoperiod and Sex Steroids. Transactions on Machine Learning and Artificial Intelligence, Vol 5 No 4 August (2017); pp: 30-42

group ( 4.72 vs. 3.26, $p<0.001$ ), however, the mean value of second acrophase was significantly advanced in GDX group than control group (14.07 vs. 15.85, $\mathrm{p}<0.001)$.

The average value of $T_{b}$ was significantly higher in GDX-T group than control group $(p<0.001,37.03$ vs. 36.78; Bonferroni t-test). The mean value of the first and second amplitude were significantly higher in control group than GDX-T one (0.94 vs. $0.87, \mathrm{p}<0.001 ; 2.50$ vs. $2.46, \mathrm{p}<0.001)$. The first acrophase was significantly delayed in GDX-T group than control group (5.15 vs. 3.26, $p<0.001)$. In the contrary, the second acrophase in GDX-T group was phase-advanced than in control group (15.22 vs. 15.85, $\mathrm{p}<0.001)$.

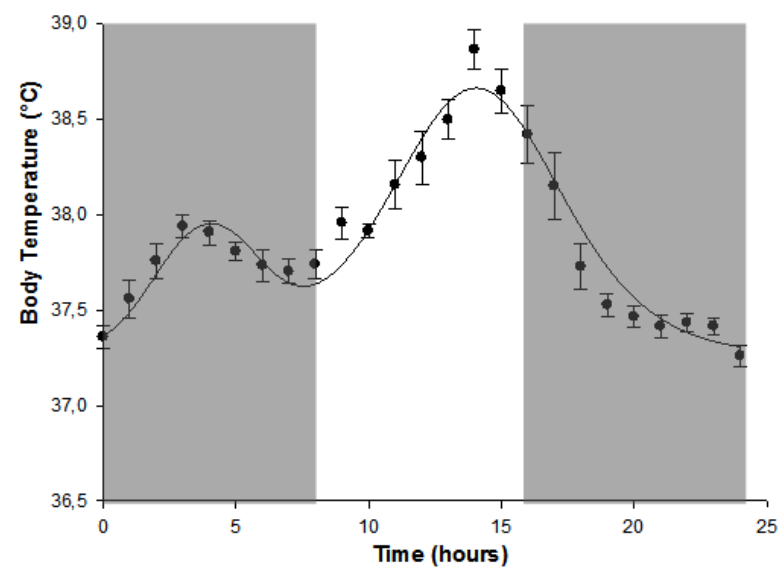

(a)

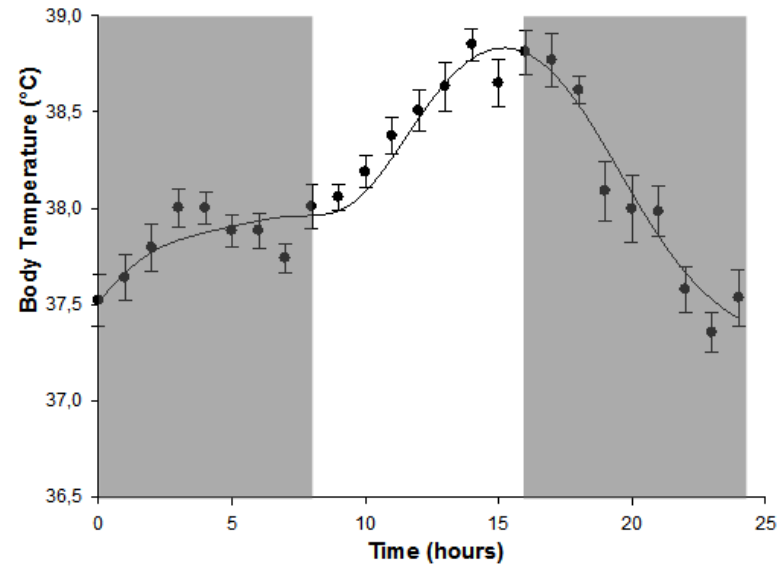

(b)

Figure 3. Daily profiles of the average of Tb rhythm in males Lemniscomys barbarus under a short photoperiod condition (LD 8:16), castration (a) and testosterone administration (b). GDX and SP GDX-T. GDX, castrated animals and $\mathrm{T}$, testosterone. The grey shading indicates the dark phase.

\subsection{Daily torpor phenomenon occurrence}

To determine if Lemniscomys barbarus engage in daily torpor phenomenon, data of continuous $T_{b}$ recording in males exposed to SP: $8 / 16$ conditions were minutely analysed between the 8 th and the 25th weeks. In the present experimental conditions, no evidence of torpor bouts occurrence was observed. No sign of hibernation bouts was either observed, even in castrated animals. The $T_{b}$ variations do not reach the characteristic values of daily torpor in small rodents $\left(15^{\circ}-20^{\circ} \mathrm{C}\right.$; [44]).

\section{Discussion}

By the use of miniature temperature-sensitive data loggers we were able to record continuously the $T_{b}$ over several months. We confirm the diurnal feature of Lemniscomys barbarus already observed with wheel-running activity parameter [21]. We observed a strong rhythm of $\mathrm{T}_{\mathrm{b}}$ in both photoperiodic conditions tested (LP and SP) in males. No arrhythmic individuals within our species can be reported, $T_{b}$ was always rhythmic and diurnal under our laboratory conditions. A daily peak of $T_{b}$ is observed during the light-phase that is logical for diurnal. Body temperature and locomotor activity rhythms are known to be robustly associated in time [38; 49]. Thanks to the determination of the profile of the daily $T_{b}$ rhythm we were able to confirm the bimodal character of the daily rhythmicity in Lemniscomys barbarus, a character also already demonstrated in report to its wheel-running activity [21]. We know, indeed, that the bimodality is very close to the locomotor activity and the corticosterone rhythms [36; 52]. In our 
animal model the daily $\mathrm{T}_{\mathrm{b}}$ rhythm was biphasic independently of photoperiodic regime, i.e. LP or SP. Moreover, the biphasic behavior seems to be a genetically strong character in Lemniscomys barbarus since it doesn't alter by the gonads status.

Many authors explain the bimodal character in terms of ecophysiology by moving forward that this mode allows the animal to avoid the important hot temperatures of the middle-day. Similar bimodal activity was obtained in other diurnal species, i.e. Arvicanthis ansorgei [6], Arvicanthis niloticus [50] and Octodon degus [31; 37]. However, in other diurnal species as Spermophilus tridecemlineatus, Canis familiaris, Equus caballus and humans $[40 ; 24 ; 34]$, the rhythm of $T_{b}$ is unimodal even if the minimal values are registered during dark-phase as for L. barbarus.

This work demonstrates that daily $T_{\mathrm{b}}$ pattern is synchronized by the light-dark cycle and that $L$. barbarus shows a flexibility of this rhythm towards photoperiod variations; this result confirms the photoperiodic character of this species [21]. A study of $c$-fos expression rhythm shows indeed that the adjustment of the functioning of the endogenous clock is fast in long compared to short days [48; 54]. It would be interesting to verify, in our animal model, the profile of the daily rhythm of the core body temperature after a bilateral destruction of the SCN to confirm the role of circadian pacemaker of these nuclei. Furthermore, many studies were interested on the seasonality of the $T_{b}$ rhythm $[57 ; 1 ; 58]$ and the influence of photoperiod via its hormone the melatonin on the mechanisms of regulation of $\mathrm{T}_{\mathrm{b}}$ was demonstrated; for example, in Acomys russatus, another diurnal species in whom the percentage of the $T_{b}$ rhythmicity is lesser in SP than in LP and the exogenous melatonin has a repressor effect on this rhythm $[16 ; 17]$.

In this work we show that the $\mathrm{T}_{\mathrm{b}}$ values oscillate between $36.41^{\circ}-39.91^{\circ} \mathrm{C}$ in males whatever the photoperiodic regime. These changes are almost similar to others endotherms who display a daily oscillation in $T_{b}$ [26]. This relatively large range of $T_{b}$ rhythm oscillations may have beneficial effect on thermoregulation in an environment with daily ambient temperature cycles like at Tétouan latitude (North of Morocco). Nevertheless, the range of $\mathrm{T}_{\mathrm{b}}$ oscillation obtained in Lemniscomys barbarus was slightly higher compared with that of a dark-active rodent like the Syrian hamster, e.g., $2.9^{\circ} \mathrm{C}$ [39]. Barbary striped grass mouse is a very lively and very active rodent as demonstrated by Lahmam (2008) [21] thanks to the wheel-running activity and the accentuate range of $T_{b}$ oscillations can be due to an increased activity as confirmed in several species [38]. Besides, in the Djungarian hamster, Phodopus sungorus, a similar-sized rodent than our model animal, mean $\mathrm{T}_{\mathrm{b}}$ was $33.18^{\circ} \mathrm{C} \pm 1.4$ in the warm $\left(20.0^{\circ} \mathrm{C} \pm 0.86\right)$ [51].

In our diurnal rodent, Lemniscomys barbarus, photoperiodic effects on the daily $T_{b}$ rhythm were observed. The amplitude of the daily $T_{b}$ rhythm was higher in LP than in SP conditions. In the Siberian hamster, a similar-sized rodent, several daily rhythms were also lower in amplitude in short days than in long ones [35].

Concerning the sexual steroids action we observed that testosterone affects the acrophase timing (phase advance peak), the amplitude and the average of the $T_{b}$ rhythm. In both group, GDX and GDX-T the mean values of $T_{b}$ were higher than control group. Indeed, the first acrophase was significantly advanced in control group than GDX and GDX-T groups. However, the second acrophase was phase-delayed in control group than GDX and GDX-T groups. The effects obtained after experimental processes such as the castration or the exogenous testosterone administration, which remain artificial approaches, are contrasted by the fact that in non treated animals exposed to LP or SP conditions the spontaneous testicular activation and gonads atrophy, respectively, can be of a variable level. 
Malika Nait Daoud, Ali Ouarour; Body Temperature Patterns in Captive Barbary Striped Grass Mousse (Lemniscomysbarbarus): Influence of Photoperiod and Sex Steroids. Transactions on Machine Learning and Artificial Intelligence, Vol 5 No 4 August (2017); pp: 30-42

It was indeed reported that the testosterone plays a role especially in the organization of the daily $T_{b}$ pattern [29]. Because sexual steroids concentrations in LP are supposed constant even after 25 weeks, average $T_{b}$, the amplitude and the acrophase did not change according to time in LP. Besides, since the age of animals captured in the nature is difficult to estimate, we cannot exclude the effect of the age which can also explain the characteristics changes of the $T_{b}$ rhythm. The animal's age, indeed, can alter the circadian rhythm of $T_{b}[28 ; 1 ; 56]$.

To survive in the rigorous conditions of the bad season, small-sized heterothermic mammals develop various strategies of seasonal acclimatization among which the expression of the daily torpor [55; 14]. This annual function is essentially controlled by photoperiod and ten weeks of SP exposure in laboratory conditions are enough for leading the torpor appearance [33]. In Tétouan region, the captures of Lemniscomys barbarus being much less successful during the bad season than during the spring and summer, we strongly suspected this rodent of living in lethargy; the torpor bouts expression was then investigated.

In our experimental conditions with food ad libitum and thermoneutrality, L. barbarus did not express episodes of torpor further to a long exposure in SP conditions (SP: 8/16) in intact and castrated animals. In the other hand, the mouse Peromyscus maniculatus is sexually inactive when the food becomes rare [3]. So, the daily torpor phenomenon in certain species of mammals can be led by a food deprivation [47]. In this work, we tried to highlight daily torpor while animals were in condition of food in excess. It is possible that in Lemniscomys barbarus a partial hardship of the food could potentiate the effect of torpor induction by SP. It's well know indeed that in temperate regions heterothermic regulations are most pronounced during long and severe winters when low ambient temperatures are normally associated with reduced food availability [13]. In future works, it would be of a certain interest to verify this track in Lemniscomys barbarus maintained in SP conditions associated to cold and food restriction.

\section{Conclusion}

This study showed for the first time the profile of daily $T_{b}$ rhythm in Lemniscomys barbarus, characterized by a strong rhythm in both photoperiodic conditions (LP and SP) with a daily peak during the light phase which confirms the diurnal feature of our species.

According to the experimental conditions of this work, we were not able to establish if Lemniscomys barbarus engage on the phenomenon of daily torpor. Nevertheless, this characteristic mechanism of energy saving in the small-sized mammals could be demonstrated in a future study by the combined effects of SP, low ambient temperature and reduction of food access.

\section{ACKNOWLEDGMENT}

The thanks of the authors are sent to Pr. Mohammed Errami, Tétouan, Morocco, for all the efforts he displayed to provide the equipment required for the animal facilities. The authors sent deeply thanks for their valuable contribution to Dr. Ibtissam Chakir from our laboratory of biology and health, faculty of sciences, Tétouan, and Dr. Patrick Vuillez from the Institute for Integrative Cellular and Neurosciences, UPR 3212 University of Strasbourg CNRS, Strasbourg, France. 


\section{REFERENCES}

[1] Benstaali C., Bogdan A., Touitou Y., 2002. Effect of a short photoperiod on circadian rhythms of body temperature and motor activity in old rats. Pflugers Arch. 444(1-2):73-79.

[2] Biggar K.K., Wu C.W., Tessier S.N., Zhang J., Pifferi F., Perret M., Storey K.B., 2015. Primate Torpor: Regulation of Stress-activated Protein Kinases During Daily Torpor in the Gray Mouse Lemur, Microcebus murinus. Genomics Proteomics Bioinformatics. 13:81-90.

[3] Blank J.L., Desjardins C., 1984. Spermatogenesis is modified by food intake in mice. Biol. Reprod. 30: 410415.

[4] Challet E., Pitrosky B., Sicard B., Malan A., Pévet P., 2002. Circadian organization in a diurnal rodent, Arvicanthis ansorgei Thomas 1910: chronotypes, responses to constant lighting conditions and photoperiodic changes. J. Biol. Rhythms. 17:52-64.

[5] Chakir I., 2014. Rôle du gène horloge Clock dans l'intégration de la photopériode par les noyaux suprachiasmatiques chez un rongeur nocturne, Mesocricetus auratus, et dans le déterminisme de la diurnalité chez un rongeur diurne, Lemniscomys barbarus. Doctoral thesis at the Abdelmalek Essaâdi University, Tétouan, Morocco.

[6] Cuesta M., Clesse D., Pévet P., Challet E., 2009. From daily behavior to hormonal and neurotransmitters rhythms: Comparison between diurnal and nocturnal rat species. Hormones and Behavior. 55: 338-347.

[7] Dardente H., Menet J.S., Challet E., Tournier B.B., Pévet P., Masson-Pévet M., 2004. Daily and circadian expression of neuropeptides in the suprachiasmatic nuclei of nocturnal and diurnal rodents. Mol. Brain. Res. 124:143-151.

[8] Dzal Y.A., Brigham R.M., 2013. The tradeoff between torpor use and reproduction in little brown bats (Myotis lucifugus). J. Comp. Physiol.B. 183 : 279-288.

[9] El Allali K., Achaâban M.R., Bothorel B., Piro M., Bouâouda H., El Allouchi M., Ouassat M., Malan A., Pévet P., 2013. Entrainment of the circadian clock by daily ambient temperature cycles in the camel (Camelus dromedarius). Am J Physiol Regul Integr Comp Physiol; 304(11):R1044-52.

[10] El Ouazzani S., Janati I.A., Magoul R., Pévet P., Saboureau M., 2011. Overwinter body temperature patterns in captive jerboas (Jaculis orientalis): Influence of sex and group. J. Comp. Physiol. B. 181(2):299309.

[11] Freeman M.E., Crissma J.K., Low G.N., Butcher R.L., Inskeep E.K., 1970. Thermogenic action of progesterone in the rat. Endocrinology. 86: 717-720.

[12] Geiser F., 2004. Metabolic rate and body temperature reduction during hibernation and daily torpor. Annu. Rev. Physiol. 66: 239-274.

[13] Geiser F., Ruf T., 1995. Hibernation versus daily torpor in mammals and birds: physiological variables and classification of torpor patterns. Physiol. Zool. 68:935-66. 
Malika Nait Daoud, Ali Ouarour; Body Temperature Patterns in Captive Barbary Striped Grass Mousse (Lemniscomysbarbarus): Influence of Photoperiod and Sex Steroids. Transactions on Machine Learning and Artificial Intelligence, Vol 5 No 4 August (2017); pp: 30-42

[14] Geiser F., Turbill C., 2009. Hibernation and daily torpor minimize mammalian extinctions. Naturwissenschaften .96:1235-1240.

[15] Golombek D.A., Rosenstein R.E., 2010. Physiology of circadian entrainment. Physiol. Rev. 90:1063-1102.

[16] Haim A., Zizapel N., 1994. Daily rhythm of body temperature in coexisting rodents of the Genus Acomys acclimated to long-photoperiod: effects of ethanol and melatonin. Thermal Balance in Health and Disease APS. 22:191-196.

[17] Haim A., Shachaf K., Zisapel N., Reiter R.J., 1997. Daily rhythm of body temperature in Acomys russatus: Their response to photoperiod manipulations and melatonin. J. therm. Biol. 22( 3): 219-222.

[18] Heldmaier G., Steinlechner S., 1981. Seasonal pattern and energetic of short daily torpor in the Djungarian hamster, Phodopus sungorus. Oecologia .48 : 265-270.

[19] Jefimow M., Ostrowski M., Jakubowska A., Wojciechowski M.S., 2014. The effects of Dietary Cholesterol on metabolism and Daily Torpor Patterns in Siberian Hamsters. Physiological and Biochemical Zoology. 87(4): 527-538.

[20] Kirsch R., Ouarour A., Pévet P., 1991. Daily torpor in the Djungarian hamster (Phodopus sungorus): photoperiodic regulation, characteristics and circadian organization. J. Comp. Physiol. A. 168:121-128.

[21] Lahmam M., El M'rabet A., Ouarour A., Pévet P., Challet E., Vuillez P., 2008. Daily behavioral rhythmicity and organization of the suprachiasmatic nuclei in the diurnal rodent, Lemniscomys barbarus. Chronobiol Int. $25: 882-904$.

[22] Lahmam M., 2008. Rythmicité comportementale journalière et organisation des noyaux suprachiasmatiques et de la rétine chez Lemniscomys barbarus, un rongeur diurne à reproduction saisonnière. Doctoral Thesis at the Abdelmalek Essaâdi University, Tétouan, Morocco.

[23] Lee T.M., Labyak S.E., 1997. Free-running rhythms and light- and dark-pulse phase response curves for diurnal Octodon degus (Rodentia). Am. J. Physiol. Regul. Integr. Comp. Physiol. 273: R278-R286.

[24] Leproult R., Van Onderbergen A., L'hermite-Baleriaux M., Van Cauter E., Copinschi G., 2005. Phase-Shifts of 24-h rhythms of hormonal release and body temperature following early evening administration of melatonin agonist agomelatine in healthy older men. Clin, Endocrinol. (Oxf) .63:298-304.

[25] Levi O., Dayan T., Kronfeld-Schor N., 2011. Adaptive thermoregulation in Golden Spiny Mice: The Influence of Season and Food Availability on Body Temperature. Physiological an Biochemical Zoology. 84(2): 175184.

[26] Lovegrove B.G., Heldmaier G., Ruf T., 1991. Perspectives of endothermy revisited: the endothermic temperature range. J Therm Biol .16(4): 185-197.

[27] Mahoney M., Bult A., Smale L., 2001. Phase response curve and light-induced fos expression in the suprachiasmatic nucleus and adjacent hypothalamus of Arvicanthis niloticus. J. Biol. Rhythms .16: 149162. 
Transactions on Machine Learning and Artificial Intelligence Vol 5 No 4, Aug 2017

[28] Maillox A., Benstaali C., Bogdan A., Auzéby A., Touitou Y., 1999. Body temperature and locomotor activity as marker rhythms of aging of the circadian system in rodents. Experimental Gerontology. 34(6): 733-740.

[29] Marrone B.L., Gentry R.T., Wade G.N., 1976. Gonadal Hormones and Body Temperature in Rats: Effects of Estrous Cycles, Castration and Steroid Replacement. Physiology \& Behavior. 17(3): 419-425.

[30] McDonald R.B., Day C., Carlson K., Stern J.S., Horwitz B.A. 1989. Effect of age and gender on thermoregulation. Am. J. Physiol. 257 (4 pt 2), R700-R704.

[31] McElhinny T.L., Smale L., Holeklamp K.E., 1997. Patterns of body temperature, activity, and reproductive behavior in a tropical murid rodent, Arvicanthis nioticus. Physiol. Behav. 62: 91-96.

[32] Mzilikazi, N., Lovegrove, B.G., 2002. Reproductive activity influences thermoregulation and torpor in the pouched mouse, Saccostomus campestris. J. comp. Physiol. B. 172: 7-16.

[33] Ouarour A., Kirsch R., Pévet P., 1991. Effects of temperature, steroids and castration on daily torpor in the Djungarian hamster (Phodopus sungorus). J. Comp. Physiol. A. 168:477-481.

[34] Piccione G., Caola G., Refinetti R., 2005. Temporal relationships of 21 physiological variable in horse and sheep. Com. Biochem. Physiol. A. Mol. Integr. Physiol. 142:389-396.

[35] Prendergast B.J., Tyler J. Stevenson T.J., Zucker I., 2013. Sex differences in Siberian hamster ultradian locomotor rhythms. Physiol. Behav. 0: 206-212.

[36] Refinetti R., Manaker, M., 1992. The circadian rhythm of body temperature. Physiol.Behav. 51:613-637.

[37] Refinetti R., 1996. Rhythm of body temperature and temperature selection are out of phase in a diurnal rodent, Octodon degus. Physiol. Behav. 60:959-961.

[38] Refinetti R. 1999a. Relationship between the daily rhythms of locomotor activity and body temperature in eight mammalian species. Am. J. Physiol. 277 (5pt2): R1493-R1500.

[39] Refinetti R., 1999b. Amplitude of the daily rhythm of body temperature in eleven mammalian species. J Thermal Biol. 24 (5-6): 477-481.

[40] Refinetti R., Piccione, G., 2004. Intra-and inter-individual variability in the circadian rhythm of body temperature of rats, squirrels, dogs, and horses. J. of Thermal Biology. 30:139-146.

[41] Refinetti R., 2006. Variability of diurnality in laboratory rodents. J. Comp. Physiol. A. 192(7):701-714.

[42] Refinetti, R., 2010. The circadian rhythm of body temperature. Frontiers in Bioscience. 15: 564-594.

[43] Ruf T., Geiser F., 2015. Daily torpor and hibernation in birds and mammals. Biol. Rev. 90:891-926.

[44] Ruf T., Heldmaier G., 1992. The impact of daily torpor on energy requirements in the Djungarian hamster, Phodopus sungorus. Physiol Zool 65: 994-1010.

[45] Schmidt P.T., Raju, J., Danaceau M., Murphy D.L., Berlin R.E., 2002. The effects of gender and gonadal steroids on the neuroendocrine ant temperature response to $\mathrm{m}$-chlorophenylpiperazine in leuropideinduced hypogonadism in women and men. Neuropsychopharmacology. 27800-27812. 
Malika Nait Daoud, Ali Ouarour; Body Temperature Patterns in Captive Barbary Striped Grass Mousse (Lemniscomysbarbarus): Influence of Photoperiod and Sex Steroids. Transactions on Machine Learning and Artificial Intelligence, Vol 5 No 4 August (2017); pp: 30-42

[46] Smale L., Lee T., Nunez A.A., 2003. Mammalian diurnality: some facts and gaps. J. Biol. Rhythms. 18:356366.

[47] Steinlechner S., Heldmaier G., Weber C., Ruf T., 1986. Role of photoperiod: pineal gland interaction in torpor control. In Heller H.C. et al. (Eds), Living in the cold: 301-307.

[48] Sumova A., Travnickova Z., Illnerova H., 1995. Memory on long but not on short days is stored in the rat suprachiasmatic nucleus. Neurosci. Lett. 200(3): 191-4.

[49] Tachinardi P., Bicudo J.E.W., Oda G.A., Valentinuzzi V.S, 2014. Rhythmic 24 h Variation of Core Body Temperature and Locomotor Activity in a Subterranean Rodent (Ctenomys aff. knighti), the Tuco-Tuco. PLOS ONE 9(1): e85674.

[50] Teresa L., McElhinny, Smale, L., Holekamp, K.E., 1997. Patterns of body temperature, Activity, and reproductive behavior in a tropical murid roden, Arvivanthis niloticus. Physiology \& Behavior. 62(1): 9196.

[51] Turbill C., Smith S., Deimel C., Ruf T., 2012. Daily torpor is associated with telomere length change over winter in Djungarian hamsters. Biol. Lett. 8:304-307.

[52] Verhagen L.A., Pévet P., Saboureau M., Sicard B., Nesme B., Claustrat B., Buijs R.M., Kalsbeek A., 2004. Temporal organization of the 24-h corticosterone rhythm in the diurnal murid rodent Arvicanthis ansorgei Thomas 1910. Brain Res. 995(2):197-204.

[53] Vitale P.M., Darrow J.M., Duncan M.J., Shustak C.A., Goldman B.D., 1985. Effects of photoperiod, pinealectomy and castration on body weight and daily torpor in Djungarian hamsters (Phodopus sungorus). J. Endocrinology. 106:367-375.

[54] Vuillez P., Jacob N., Teclemariam-Mesbah R., Pévet P., 1996. In Syrian and European hamsters, the duration of sensitive phase to light of the suprachiasmatic nuclei depends on the photoperiod. Neurosci Lett.208(1):37-40.

[55] Wang L.C.H., Wolomyk M.W., 1988. Torpor in mammals and birds. Can. J. Zool. 66: 133-137.

[56] Weinert D., 2010. Circadian temperature variation and ageing. Ageing Res. Rev. 9(1): 51-60.

[57] Wollnik, F., Schimidt, B., 1995. Seasonal and rhytms of body temperature in the European hamster (Cricetus cricetus) under semi-natural conditions. J. Comp Physiol B. 165: 171-182

[58] Zhi-Jun Zhaon, Jing Cao, Zheng-Chao Liu, Gui-Ying Wang, Lu-Sheng Li, 2010. Seasonal regulations of resting metabolic rate and thermogenesis instriped hamster (Cricetulus barabensis). J. of Thermal Biology. 35: 401-405.

[59] Zuloaga D.G., McGiverna R.F., Handa R.J., 2009. Organizational influence of the postnatal testosterone surge on the circadian rhythm of core body temperature of adult male rats. Brain Research. 1268:68-75. 